\title{
Comparative analysis of cardio metabolic profile before and after 34 weeks in Ghanaian preeclamptic patients
}

\author{
Ababio GK ${ }^{*}$, Tetteh D², Adu-Bonsaffoh K ${ }^{3}$, Morvey $D^{4}$, Botchway $F^{5}$, Abindau E ${\text {, Narh } G^{7} \text { and Quaye IKE }}^{8}$ \\ ${ }^{1}$ University of Ghana SBAHS, Department of Medical Biochemistry, Accra, Ghana \\ ${ }^{2}$ Accra Polytechnic, Department of Clinical Biochemistry, Accra, Ghana \\ ${ }^{3}$ Korle-Bu Teaching Hospital, Department of Obstetrics and Gynecology, Accra, Ghana \\ ${ }^{4}$ Clinical Documentation Improvement Specialist, Southern California Hospital, Culver City, Ca 90232 \\ ${ }^{5}$ Korle-Bu Teaching Hospital, Department of Child Health, Accra, Ghana \\ ${ }^{6}$ University of Ghana SBAHS, Department of Physiology, Accra, Ghana \\ ${ }^{7}$ University of Ghana, Department of Biochemistry, Accra, Ghana \\ 8University of Namibia School of Medicine, Department of Medical Biochemistry
}

\begin{abstract}
Background: Over the past decade there has been an increased focus on the link between the history of preeclampsia and cardio metabolic syndrome - a danger signaling event. The impact of this event is now becoming alarming in developing countries but data is scanty. Hence the need to collate information in Ghana.

Aim: To compare cardio metabolic profile of preeclampsia before and after 34 weeks in Ghana

Methodology: The nested case control study was located at the Obstetrics and Gynecology department of the Korle-Bu Teaching Hospital (KBTH). One hundred and sixty-four (164) consented subjects were recruited after ethical clearance was obtained and structured questionnaire administered to them. Four (4) ml blood and $5 \mathrm{~mL}$ urine samples were taken for biochemical analysis and urinalysis respectively. Randox automated chemistry analyzer was used to quantify blood chemistry. The data was captured as protected health information (PHI) and analyzed with SPSS version 18.

Results: In preeclampsia, increased cardio metabolic scores before term were associated with low birth weight while lactate dehydrogenase (LDH) increased before 34 weeks and rather decreased after 34 weeks. Systolic blood pressure (SBP), white blood cells (WBC) and aspartate transaminase (AST) increased as well in PE clients while platelet (PLT), low density lipoprotein (LDL) and birth weight decreased when these variables were doubly stratified by weeks of gestation and cardio metabolic profile.
\end{abstract}

Conclusion: Among preeclampsia, increased cardio metabolic profile before term were associated with low birth weight.

\section{Introduction}

Cardio metabolic syndrome [1-5] is a danger signaling [6] event that is generated from trauma and or tissue damage or cellular death resulting from lactate dehydrogenase (LDH) leakage [7] from the cell. This signaling event revolves round the interplay between metabolic factors and cytokines which activates the immune system [6] through inflammatory nutrients [8] or fatty meals $[9,10]$ or excess antiinflammatory nutrient due to dose dependent loss of specificity [8]. The metabolic factors include high blood pressure, increased insulin levels, increased body mass index (BMI), and abnormal cholesterol levels [1-5,11]. These factors independently lead to clinical array of diseases such as diabetes, stroke, cardiovascular disorders, dyslipidemia and or hypercholesterolemia [1-5,11]. The disease conditions are as a result of (i) a modification of low density lipoprotein (LDL) to oxidize - LDL (ox-LDL) [12-17] in the endothelial system leading to its impairment [18]; or (ii) induction of monocyte chemotactic protein 1 (MCP-1) by ox-LDL [19]; or (iii) recruitment of monocytes and T-cells to the intima by ox-LDL that promotes the transformation of monocytes to macrophages [16]. Ox-LDL, together with angiotensin II, TNF- $\alpha$ and or local stress also induce lectin-like ox-LDL receptor 1 (LOX-1) in the endothelial system causing its impairment [18]. A potent agonist of ox-
LDL is peroxisome proliferator-activated receptor (PPAR- $\gamma$ ), a lipid sensing ligand activated transcription factor [20-27] which binds to toll-like receptors (TLR) $[28,29]$ and initiate a cellular response leading to activation of NF- $\mathrm{kB}$ and regulation of immune responsive genes. PPAR- $\gamma$ is therefore involved in lipid metabolism, glucose homeostasis and inflammatory activity [24] through activation of NF- $\kappa$ B. Under extremely abnormal circumstances, immune responsive genes finds alternate routes thereby increasing protein catabolism (e.g. excretion of long or short polypeptide chains in urine) or increasing gluconeogenesis. Both protein catabolism and increased gluconeogenesis could affect maternal and perinatal outcomes of pregnancy.

However, it remains unclear whether pregnancy induced hypertensive women and or preeclampsia have altered levels of

Correspondence to: Ababio GK, University of Ghana SBAHS, Department of Medical Biochemistry, Accra, Ghana, E-mail: graceababio2002@yahoo.com

Key words: cardio metabolic profile, preeclampsia, pregnancy outcomes

Received: August 20, 2016; Accepted: September 26, 2016; Published: September 29, 2016 
cardio metabolic profiles before pregnancy or whether the conditions arise due to disturbances induced by the pregnancy itself. A better understanding of the underlying pathophysiology of preeclampsia $(\mathrm{PE})$ is therefore needed for early intervention. Cardiometabolic profile might provide such platform of data support, since increasing risk is seen in the pathogenesis of PE.

$\mathrm{PE}$ is a multifactorial disorder with no specific identifiable etiology and accounting for 5-7\% of all pregnancies with significant maternal and perinatal morbidity and mortality [30-31]. Most of the studies into the etiology and the pathogenesis of preeclampsia [30-32] had been conducted in the developed world with limited data in the developing countries such as Ghana. It is therefore imperative that such studies be replicated in our indigenous women to provide evidence base information to aid in the prevention and management of preeclampsia and its complications. The current research thereby seeks to investigate the role of cardio metabolic risk on the pathogenesis and outcomes of $\mathrm{PE}$ in the Ghanaian population.

Aim: To compare cardio metabolic profile of preeclampsia before and after 34 weeks in Ghana

\section{Method}

Anthropometry: A questionnaire for clinical information was obtained after informed consent and ethical review (MS-Et/M.3 P.3.2/2013 - 2014) for this nested case-control study situated at the Obstetrics and Gynecology unit, Korle-Bu teaching hospital, Accra. The sample consisted of 164 participants; fifty-five (55) controls, fiftyfive (55) pregnant normotensives and fifty-four (54) PE.

\section{Dependent variable: Cardio metabolic profile}

Independent variables: Body mass index (BMI), Age, blood pressure, creatinine, lactate dehydrogenase, week of gestation, lipid profile and full blood count (FBC).

Term pregnancy: Gestational age of 37 weeks and above

Case definition: Metabolic syndrome was defined in accordance with NCEP - ATP III [11] as the presence of three (3) or more of the following characteristics:

i. Waist circumference $>102 \mathrm{~cm}$ in men and $>88 \mathrm{~cm}$ in women

ii. Triglyceride levels $\geq 150 \mathrm{mg} / \mathrm{dl}$,

iii. High density lipoprotein cholesterol $<50 \mathrm{mg} / \mathrm{dl}$ in women

iv. Blood pressure $\geq 130 / 85 \mathrm{~mm} \mathrm{Hg}$

v. Fasting serum glucose $\geq 110 \mathrm{mg} / \mathrm{dl}$.

Cardio metabolic scoring profile: Each risk factor of cardiometabolic profile was divided into the categories shown in Table 1.

Inclusion and exclusion criteria: Only patients diagnosed with PE were recruited after obtaining their consent as cases. Control subjects included normotensive pregnant and non-pregnant women who gave informed consent. Patients with a history of renal disease, chronic hypertension, diabetes, molar pregnancy, urinary tract infection, thyroid dysfunction and infectious diseases were excluded.

Blood collection and processing: Four milliliter $(4 \mathrm{ml})$ blood was drawn from an antecubital vein by means of a plastic syringe and dispensed into EDTA (for FBC) and gel tubes taking careful precautions. Sysmex hematological auto analyzer was used to quantify FBC whilst RANDOX auto analyzer was used for the determination of lipid profile, $\mathrm{LDH}$ and creatinine levels.

Urinalysis: Five $\mathrm{mL}$ spot urine was obtained to determine proteinuria and to categorize subjects into normotensives and PE.

For the purposes of this study, PE was defined based on American College of Obstetricians and Gynecologist criteria. Accordingly, PE is defined as diastolic blood pressure of $\geq 90 \mathrm{mmHg}$ and or systolic blood pressure of $\geq 140 \mathrm{mmHg}$ with proteinuria $\geq 300 \mathrm{mg} / \mathrm{dl}$ occurring after 20 weeks of gestation.

\section{Data management}

The integrity of patient records, ethics and data protection were taken into consideration. Data were created on a spreadsheet, corrected for errors, three (3) different backups created and SPSS version 18 used for analysis.

\section{Results}

The study was completed by 164 subjects (Table 2). The ratio of pregnant normotensives to controls to $\mathrm{PE}$ was 1:1:1 respectively. Majority of the subjects were close to the study site, Accra (Table 2) with few exceptional referral PE cases from other parts of the country. All subjects attained at least some level of education while artisan formed the major occupation of these subjects. Significant statistical differences were observed in a major number of clinical variables studied except BMI (Table 3). Also, with the exception of a decreased platelet among PE clients; systolic blood pressure (SBP), diastolic blood pressure (DBP), liver enzymes, urine protein, urine $\mathrm{pH}$, white blood cells (WBC), lipids, lactate dehydrogenase (LDH) and cardio metabolic profile significantly increased. However, when some of these variables were doubly stratified by the weeks of gestation and cardio metabolic profile; SBP, WBC and aspartate transaminase (AST) increased in PE clients while platelet (PLT), low density lipoprotein (LDL) and birth weight decreased (Table 4). LDH, on the other hand, increased with lesser weeks of gestation and rather decreased after 34 weeks in PE subjects. The hypothesis that cardio metabolic profile contributes significantly to the pathogenesis and outcomes of PE was also tested (Table 4 and 5). Table 1 scoring system was used in calculating cardiometabolic profile.

\section{Discussion}

In this study, a report on the association of cardio metabolic

Table 1. Cardio metabolic profile scoring system.

\begin{tabular}{|c|c|c|c|c|c|c|c|c|c|c|c|}
\hline Age (years) & score & Smoker & score & LDL (mg/dL) & score & HDL (mg/dL) & score & TG (mg/dL) & score & SBP (mmHg) & score \\
\hline $18-22$ & 0 & No & 0 & $<100$ & 0 & $<35$ & 11 & $<100$ & 0 & $<120$ & 0 \\
\hline $23-27$ & 6 & Yes & 8 & $100-129$ & 5 & $35-44$ & 8 & $100-149$ & 2 & $120-129$ & 2 \\
\hline $28-32$ & 11 & & & $130-159$ & 10 & $45-54$ & 5 & $150-199$ & 3 & $130-139$ & 3 \\
\hline $33-37$ & 16 & Drinking & score & $160-189$ & 14 & $\geq 55$ & 0 & $\geq 200$ & 4 & $140-159$ & 5 \\
\hline $38-42$ & 21 & No & 0 & $\geq 190$ & 20 & & & & & $\geq 160$ & 8 \\
\hline $43-47$ & 26 & Yes & 8 & & & & & & & & \\
\hline
\end{tabular}

$1 \mathrm{mmol} / \mathrm{L}=18 \mathrm{mg} / \mathrm{dL} ; \mathrm{SBP}=$ systolic blood pressure; $\mathrm{DBP}=$ diastolic blood pressure; HDL=high density lipoprotein; $\mathrm{LDL}=$ low density lipoprotein; TG=triglyceride 
Table 2. Study demographics.

\begin{tabular}{|c|c|c|c|}
\hline & Preg. Normotensive [55] & Control [55] & Preeclampsia [54] \\
\hline $\begin{array}{l}\text { Residing in } \\
\text { Greater Accra } \\
\text { Central } \\
\text { Other }\end{array}$ & $\begin{array}{c}49(89.0)^{*} \\
6(10.9)\end{array}$ & $\begin{array}{c}50(90.9) \\
5(9.1)\end{array}$ & $\begin{array}{c}25(46.3) \\
5(9.3) \\
24(44.4)\end{array}$ \\
\hline $\begin{array}{l}\text { Tribe } \\
\text { Akan } \\
\text { Ga } \\
\text { Ewe } \\
\text { Frafra } \\
\text { Fante } \\
\text { No answer }\end{array}$ & $\begin{array}{c}25(45.5) \\
13(23.6) \\
8(14.5) \\
4(7.3) \\
5(9.1)\end{array}$ & $\begin{array}{c}5(9.1) \\
32(58.2) \\
5(9.1) \\
6(10.9) \\
3(5.5) \\
4(7.3)\end{array}$ & $\begin{array}{c}15(27.8) \\
6(11.1) \\
7(13.0) \\
5(9.3) \\
1(1.9) \\
20(37.0)\end{array}$ \\
\hline $\begin{array}{l}\text { Education } \\
\text { Primary } \\
\text { Junior } \\
\text { Senior } \\
\text { Tertiary } \\
\text { No answer }\end{array}$ & $\begin{array}{c}6(10.9) \\
14(25.5) \\
14(25.5) \\
21(38.2)\end{array}$ & $\begin{array}{c}6(10.9) \\
21(38.2) \\
16(29.1) \\
13(23.6)\end{array}$ & $\begin{array}{c}9(16.7) \\
10(18.5) \\
9(16.7) \\
4(7.4) \\
22(40.7)\end{array}$ \\
\hline $\begin{array}{l}\text { Occupation } \\
\text { Artisan } \\
\text { Nurse } \\
\text { Housewife } \\
\text { Banker } \\
\text { Student } \\
\text { Teacher } \\
\text { No answer } \\
\text { Unemployed }\end{array}$ & $\begin{array}{c}34(61.8) \\
6(10.9) \\
4(7.3) \\
2(3.6) \\
2(3.6) \\
7(12.7)\end{array}$ & $\begin{array}{c} \\
8(14.5) \\
4(7.3) \\
9(16.4)\end{array}$ & $\begin{array}{c}25(46.3) \\
2(3.7) \\
\\
\\
1(1.85) \\
24(44.4) \\
2(3.7)\end{array}$ \\
\hline
\end{tabular}

* Number $(\%)$

Table 3. Clinical variable in the study.

\begin{tabular}{|c|c|c|c|c|c|c|c|}
\hline & Pregnant normotensive & Control & Preeclampsia & ANOVA p-value & $\begin{array}{l}\text { Preg. Normo x } \\
\text { control (p-value) }\end{array}$ & $\begin{array}{c}\text { PE } x \text { control } \\
\text { (p-value) }\end{array}$ & $\begin{array}{c}\text { PE x preg. Normo } \\
\text { (p-value) }\end{array}$ \\
\hline Age (years) & $27.87 \pm 4.21(\mathbf{5 5})^{¥}$ & $34.50 \pm 8.79(\mathbf{5 5})$ & $30.95 \pm 6.00(40)$ & 0.000 & 0.000 & 0.030 & 0.004 \\
\hline BMI $\left(\mathrm{kg} / \mathrm{m}^{2}\right)$ & $29.40 \pm 6.40(\mathbf{5 5})$ & $27.60 \pm 6.32(\mathbf{5 5})$ & $28.90 \pm 6.30(32)$ & 0.318 & 0.141 & 0.357 & 0.725 \\
\hline SBP (mmHg) & $108.65 \pm 17.65(\mathbf{5 5})$ & $108.67 \pm 21.20(48)$ & $139.80 \pm 33.80(42)$ & 0.000 & 0.996 & 0.000 & 0.000 \\
\hline DBP (mmHg) & $69.76 \pm 12.67(\mathbf{5 5})$ & $70.50 \pm 12.63(48)$ & $90.20 \pm 26.00(42)$ & 0.000 & 0.768 & 0.000 & 0.000 \\
\hline $\begin{array}{l}\text { Alanine } \\
\text { aminotransferase (U/L) }\end{array}$ & $6.11 \pm 3.40(53)$ & $15.87 \pm 5.36(\mathbf{5 5})$ & $12.37 \pm 17.32(47)$ & 0.000 & 0.000 & 0.158 & 0.011 \\
\hline $\begin{array}{l}\text { Aspartate } \\
\text { aminotransferase (U/L) }\end{array}$ & $13.02 \pm 3.87(53)$ & $24.67 \pm 9.07(\mathbf{5 5})$ & $39.18 \pm 33.71(47)$ & 0.000 & 0.000 & 0.003 & 0.000 \\
\hline Urine protein & $10.64 \pm 7.04(\mathbf{5 5})$ & $7.07 \pm 5.58(53)$ & $163.90 \pm 124.90(33)$ & 0.000 & 0.004 & 0.000 & 0.000 \\
\hline Urine $\mathbf{p H}$ & $5.80 \pm 0.85(\mathbf{5 5})$ & $5.40 \pm 0.79(53)$ & $6.04 \pm 0.824(33)$ & 0.002 & 0.013 & 0.001 & 0.198 \\
\hline Specific gravity & $1.01 \pm 0.01(\mathbf{5 5})$ & $1.02 \pm 0.01(53)$ & $1.02 \pm 0.05(33)$ & 0.323 & 0.000 & 0.773 & 0.464 \\
\hline WBC $(\mathbf{x 1 0} / \mathbf{L})$ & $7.80 \pm 1.63(\mathbf{5 5})$ & $5.73 \pm 1.42(53)$ & $9.40 \pm 3.90(43)$ & 0.000 & 0.000 & 0.000 & 0.007 \\
\hline $\operatorname{RBC}\left(\times 10^{12} / L\right)$ & $4.00 \pm 0.47(\mathbf{5 5})$ & $4.64 \pm 0.39(53)$ & $4.70 \pm 1.50(43)$ & 0.000 & 0.000 & 0.780 & 0.002 \\
\hline PLT $\left(x 10^{9} / \mathrm{L}\right)$ & $242.00 \pm 70.80(\mathbf{5 5})$ & $297.45 \pm 82.10(53)$ & $221.40 \pm 93.20(43)$ & 0.000 & 0.000 & 0.000 & 0.217 \\
\hline$H b(g / d L)$ & $11.00 \pm 2.39(\mathbf{5 5})$ & $12.98 \pm 1.3853)$ & $12.40 \pm 3.10(43)$ & 0.000 & 0.000 & 0.225 & 0.013 \\
\hline Creatinine $(\mu \mathrm{mol} / \mathrm{L})$ & $52.60 \pm 17.30(40)$ & $68.80 \pm 10.95(53)$ & $64.20 \pm 20.80(49)$ & 0.000 & 0.000 & 0.161 & 0.006 \\
\hline Cholesterol (mmol/L) & $5.23 \pm 1.20(40)$ & $4.50 \pm 1.22(53)$ & $5.52 \pm 1.40(\mathbf{5 4})$ & 0.000 & 0.005 & 0.000 & 0.295 \\
\hline Triglycerides (mmol/L) & $1.88 \pm 0.63(40)$ & $0.72 \pm 0.43(53)$ & $2.00 \pm 0.96(\mathbf{5 4})$ & 0.000 & 0.000 & 0.000 & 0.493 \\
\hline HDL (mmol/L) & $1.25 \pm 0.37(40)$ & $0.95 \pm 0.40(53)$ & $1.51 \pm 0.42(\mathbf{5 4})$ & 0.000 & 0.000 & 0.000 & 0.002 \\
\hline LDL (mmol/L) & $4.10 \pm 1.17(40)$ & $3.08 \pm 1.20(53)$ & $3.70 \pm 1.50(\mathbf{5 4})$ & 0.001 & 0.000 & 0.020 & 0.165 \\
\hline LDH (U/L) & $232.10 \pm 68.30(40)$ & $346.20 \pm 145.40(50)$ & $307.90 \pm 160.50(49)$ & 0.000 & 0.000 & 0.216 & 0.007 \\
\hline Cardiometabolic profile & $19.70 \pm 3.50(40)$ & $17.30 \pm 9.40(53)$ & $21.00 \pm 8.50(54)$ & 0.051 & 0.128 & 0.035 & 0.365 \\
\hline
\end{tabular}

* Mean \pm standard deviation (number); $\mathrm{BMI}=$ body mass index; $\mathrm{SBP}=$ systolic blood pressure; $\mathrm{DBP}=$ diastolic blood pressure; $\mathrm{WBC}=$ white blood cell; $\mathrm{RBC}=$ red blood cell; $\mathrm{PLT}=$ platelet $\mathrm{Hb}=$ hemoglobin; $\mathrm{HDL}=$ high density lipoprotein; $\mathrm{LDL}=$ low density lipoprotein; $\mathrm{LDH}=$ lactate dehydrogenase

profile 'before and after' 34 weeks and adverse pregnancy outcomes were presented. We found significantly increased cardio metabolic profile before term was associated with low birth weight. We have also formulated a sensitive but simple method of calculating cardio metabolic profile to aid clinicians in assessment of future risk of developing the syndrome in PE. This study adds unto the existing knowledge elsewhere that the relationship between cardio metabolic scores and adverse pregnancy outcomes were inversely related [33,34].

Barden and others [35] also suggested that predisposition to metabolic syndrome sensitized women to develop preeclampsia. Even though the present study depicted this trend, lack of extensive patient follow-ups and their relocation and or moving back to their various destinations affected further sampling parameters. Notwithstanding, data from Norwegian research indicated a two-fold and four-fold increase of PE with body mass index (BMI $\left.>27.08 \mathrm{~kg} / \mathrm{m}^{2}\right)$ and blood pressure (SBP $>121 \mathrm{mmHg}$ and $\mathrm{DBP}>71 \mathrm{mmHg}$ ) respectively [36]. A meta-analysis had also confirmed the relationship of hypertension to lower birth weight and head circumference, as well as the importance 
Table 4. Cardio metabolic profile, weeks of gestation and some indicators.

\begin{tabular}{|c|c|c|c|c|c|c|c|c|c|c|c|c|}
\hline \multirow[t]{3}{*}{$\mathbf{s}$} & \multicolumn{6}{|l|}{$20 \leq x<34$ weeks } & \multicolumn{6}{|l|}{$x \geq 34$ weeks } \\
\hline & \multicolumn{2}{|l|}{$<20$ cardiomet score } & \multirow[t]{2}{*}{$P$-value } & \multicolumn{3}{|c|}{$>20$ cardiomet score } & \multicolumn{2}{|l|}{$<20$ cardiomet score } & \multirow[t]{2}{*}{$P$-value } & \multicolumn{3}{|c|}{$>20$ cardiomet score } \\
\hline & Preg. normo & $\mathbf{P E}$ & & Preg. normo & PE & $P$-value & Preg. normo & PE & & Preg. normo & PE & $P$-value \\
\hline Gravidity & $2.48 \pm 1.47(23)$ & $2.00 \pm 1.41(7)$ & 0.452 & $2.31 \pm 1.54(13)$ & $3.4 \pm 0.89(12)$ & 0.043 & $2.52 \pm 1.63(21)$ & $3.33 \pm 1.15(4)$ & 0.356 & $1.89 \pm 1.27(9)$ & $2.50 \pm 2.12(11)$ & 0.459 \\
\hline Parity & $2.26 \pm 1.39(23)$ & $1.00 \pm 1.41(7)$ & 0.046 & $0.93 \pm 1.43(14)$ & $1.40 \pm 1.34(12)$ & 0.398 & $1.33 \pm 1.53(21)$ & $2.00 \pm 1.73(4)$ & 0.439 & $0.67 \pm 0.71(9)$ & $1.50 \pm 2.12(11)$ & 0.278 \\
\hline $\begin{array}{l}\text { Birth weight } \\
\text { (g) }\end{array}$ & $2578.33 \pm 861.31(9)$ & $\begin{array}{l}1900.00 \pm \\
1272.00(7)\end{array}$ & 0.224 & $\begin{array}{l}2575.00 \pm \\
499.15(4)\end{array}$ & $750.00 \pm 353.55(2)$ & 0.010 & $2566.67 \pm 1267.54(6)$ & $2733.30 \pm 808.3(3)$ & 0.844 & $\begin{array}{l}3000.00 \pm 346.41 \\
\text { (3) }\end{array}$ & - & - \\
\hline $\begin{array}{l}\text { SBP } \\
(\mathrm{mmHg})\end{array}$ & $109.65 \pm 16.81(19)$ & $\begin{array}{l}135.60 \pm 34.45 \\
(7)\end{array}$ & 0.016 & $\begin{array}{l}95.43 \pm 28.67 \\
(14)\end{array}$ & $154.5 \pm 22.29(10)$ & 0.000 & $113.65 \pm 15.91(17)$ & $143.0 \pm 37.64(4)$ & 0.021 & $166.19 \pm 27.69(9)$ & $163.3 \pm 23.98(9)$ & 0.815 \\
\hline $\begin{array}{l}\text { LDH } \\
\text { (U/L) }\end{array}$ & $230.50 \pm 89.08(6)$ & $\begin{array}{l}381.63 \pm 185.16 \\
(7)\end{array}$ & 0.096 & $\begin{array}{l}235.08 \pm 65.34 \\
(14)\end{array}$ & $\begin{array}{l}370.14 \pm 158.62 \\
(12)\end{array}$ & 0.007 & $201.60 \pm 65.22(5)$ & $207.97 \pm 5.46(4)$ & 0.853 & $\begin{array}{l}477.91 \pm 709.95 \\
(9)\end{array}$ & $\begin{array}{l}220.96 \pm 93.34 \\
(10)\end{array}$ & 0.271 \\
\hline \begin{tabular}{|l|} 
LDL \\
$(\mathrm{mmol} / \mathrm{L})$
\end{tabular} & $3.78 \pm 0.78(6)$ & $2.88 \pm 0.89(7)$ & 0.081 & $4.58 \pm 1.00(14)$ & $3.17 \pm 1.16(12)$ & 0.002 & $3.91 \pm 0.89(5)$ & $4.20 \pm 0.78(4)$ & 0.625 & $3.94 \pm 1.49(9)$ & $2.92 \pm 1.17(10)$ & 0.113 \\
\hline $\begin{array}{l}\text { WBC } \\
\left(\mathbf{x} 10^{9}\right)\end{array}$ & $8.27 \pm 1.56(22)$ & $8.34 \pm 3.55(7)$ & 0.941 & $8.15 \pm 2.91(13)$ & $10.81 \pm 4.82(10)$ & 0.115 & $7.72 \pm 1.19(21)$ & $7.79 \pm 2.80(4)$ & 0.933 & $7.37 \pm 2.18(9)$ & $8.23 \pm 4.12(9)$ & 0.587 \\
\hline $\begin{array}{l}\text { PLT } \\
\left(\mathbf{x 1 0} 0^{9}\right)\end{array}$ & $248.72 \pm 78.92(22)$ & $\begin{array}{l}188.83 \pm 49.10 \\
(7)\end{array}$ & 0.071 & $\begin{array}{l}248.85 \pm 59.24 \\
(13)\end{array}$ & $279.10 \pm 86.47(10)$ & 0.330 & $239.38 \pm 72.26(21)$ & $192.00 \pm 104.06(4)$ & 0.272 & $262.44 \pm 84.25(9)$ & $\begin{array}{l}202.89 \pm 82.78 \\
\text { (9) }\end{array}$ & 0.149 \\
\hline $\begin{array}{l}\text { AST } \\
(\mathrm{U} / \mathrm{L})\end{array}$ & $12.15 \pm 2.84(17)$ & $\begin{array}{l}38.38 \pm 31.61 \\
(7)\end{array}$ & 0.002 & $13.14 \pm 4.31(7)$ & $56.44 \pm 53.61(12)$ & 0.050 & $13.72 \pm 4.64(11)$ & $26.93 \pm 18.08$ & 0.000 & $15.53 \pm 6.09(8)$ & $\begin{array}{l}44.79 \pm 28.81 \\
(10)\end{array}$ & 0.012 \\
\hline
\end{tabular}

cardiomet $=$ cardio metabolic; AST=aspartate dehydrogenase

Table 5. Cardio metabolic profile and birth weights from PE subjects.

\begin{tabular}{|l|c|c|}
\hline & Birth weight $(\mathbf{g})$ & \\
\hline$<\mathbf{2 0}$ cardiomet score & $2220.00 \pm 978.43(10)$ & \multirow{2}{*}{$\mathrm{t}_{10,724.2}=2.03$} \\
\hline $\mathbf{2 0}$ cardiomet score & $750.00 \pm 353.55(2)$ & \\
\hline p-value & 0.0698 & \\
\hline
\end{tabular}

of catch-up growth [37]. However, when this same type of analysis were restricted to studies with published correlations between systolic $\mathrm{BP}$ and birth weight, the association was not so strong; an indication of other eminent factors $[1-5,11]$ in the environ which could possibly cause such effects $[37,38]$.

There is also evidence outside of pregnancy that increased leukocyte counts within normal range were associated with metabolic disorders in women $[39,40]$. Other findings suggested that slightly increased leukocyte counts may modestly be associated with the development of pregnancy induced hypertensive disorder. However, leukocyte count alone was relatively a non-specific marker of inflammation due to it being influenced by other factors such as infection and certain medications [41]. Data from Kaiser Permanente MHC also showed that inflammation as measured by increased leukocytes was predictive of hypertension but were unable to present the potential mechanism $[42,43]$. We are by this study suggesting that preeclampsia (PE), through the cardio metabolic signaling pathway was as a result of the modification of LDL to form ox-LDL which did trigger foam cell formation. This was because of the relatively low LDL levels observed. Current literature also suggested that upon activation of macrophage system (foam cells) by ox-LDL [12-19], primary mediators like cytokines or chemokines elicited the recruitment of secondary mediators e.g. white blood cells (WBC) to thus impair the endothelial system.

Despite the well-intentioned efforts at making laboratory diagnosis of PE and it complications especially HELLP syndrome (Haemolysis, Elevated liver enzymes and low platelets) based on the presence of hemolysis, elevations of liver enzymes (AST, ALT), LDH and proteinuria, substantial inter-laboratory differences remain a major problem. This was partly due to the cutoff point or reference ranges used to establish an abnormal test and the different number of assays used to measure these tests. In this study, the standardized hospital protocol was used to ascertain the various parameters.

\section{Conclusion}

In preeclampsia, increased cardio metabolic profiles before term were associated with low birth weight.

\section{Acknowledgement}

This work was supported by grants from ORID, University of Ghana, Legon. The authors duly acknowledge Mensah J, Amponsah C, staff of Obstetrics and Gynecology of the Korle-Bu Teaching Hospital, Accra, Ghana; and also, Medical Biochemistry, Univ. of Ghana SBAHS.

\section{Funding disclosure}

Work from the authors' laboratory was supported by grants from ORID, University of Ghana, Legon.

The authors declare that there is no conflict of interest.

\section{References}

1. Castro JP, El-Atat FA, McFarlane SI, Aneja A, Sowers JR (2003) Cardio metabolic syndrome: pathophysiology and treatment. Current Hypertension Reports 5: 393-401. [Crossref]

2. Sowers JR (2001) Update on the cardiometabolic syndrome. Clin Cornerstone 4: 17 23. [Crossref]

3. Kirk EP, Klein S (2009) Pathogenesis and pathophysiology of the cardiometabolic syndrome. J Clin Hypertens (Greenwich) 11: 761-765. [Crossref]

4. Bray MS, Tsai JY, Villegas-Montoya C, Boland BB, Blasier Z, et al.(2010) Time-ofday-dependent dietary fat consumption influences multiple cardio metabolic syndrome parameters in mice. International Journal of Obesity 34:1589-1598. [Crossref]

5. Dominguez LJ, Barbagallo M (2007) The cardiometabolic syndrome and sarcopenic obesity in older persons. J Cardiometab Syndr 2: 183-189. [Crossref]

6. Mathieu P, Pibarot P, Després JP (2006) Metabolic syndrome: the danger signal in atherosclerosis. Vasc Health Risk Manag 2: 285-302. [Crossref]

7. Peralta PM, Basavilvazo RM, Cruz AA, Sánchez AS, Guzmán IM, et al. (2004) Clinical signi?cance of the laboratory determinations in preeclamptic patients. Ginecol Obstet Mex 72: 57-62. [Crossref]

8. Gregor MF, Hotamisligil GS (2011) Inflammatory mechanisms in obesity. Annu Rev Immunol 29: 415-445. [Crossref]

9. Cani PD, Amar J, Iglesias MA, Poggi M, Knauf C, et al. (2007) Metabolic endotoxemia initiates obesity and insulin resistance. Diabetes 56: 1761-1772. [Crossref]

10. Erridge C, Attina T, Spickett CM, Webb DJ (2007) A high-fat meal induces lowgrade endo toxemia: evidence of a novel mechanism of postprandial inflammation. The 
American Journal of Clinical Nutrition. 86: 1286-1292. [Crossref]

11. NCEP-ATP III (2001) Executive summary of the Third Report of The National Cholesterol Education Program (NCEP) Expert Panel on Detection, Evaluation, and Treatment of High Blood Cholesterol in Adults (Adult Treatment Panel III). JAMA 285: 2486-2497.

12. Michelsen KS, Doherty TM, Shah PK, Arditi M (2004) TLR signaling: an emerging bridge from innate immunity to atherogenesis. J Immunol 173: 5901-5907. [Crossref]

13. Holvoet P1 (2004) Oxidized LDL and coronary heart disease. Acta Cardiol 59: 479484. [Crossref]

14. Ylä-Herttuala S, Palinski W, Rosenfeld ME, Parthasarathy, Carew SA, et al. (1989) Evidence for the presence of oxidatively modified low density lipoprotein in atherosclerotic lesions of rabbit and man. J Clin Invest 84: 1086-1095. [Crossref]

15. Shaw PX (2004) Rethinking oxidized low-density lipoprotein, its role in atherogenesis and the immune responses associated with it. Arch Immunol Ther Exp (Warsz) 52: 225239.

16. Weber C, Erl W, Weber PC (1995) Enhancement of monocyte adhesion to endothelia cells by oxidatively modified low-density lipoprotein is mediated by activation of CD11b. Biochem Biophys Res Commun 206: 621-628. [Crossref]

17. Navab M, Berliner JA, Watson AD, Hama SY, Territo, et al. (1996) The Yin and Yang of oxidation in the development of the fatty streak. A review based on the 1994 George Lyman Duff Memorial Lecture. Arterioscler Thromb Vasc Biol 16: 831-842. [Crossref]

18. Sawamura T, Kume N, Aoyama T, Moriwaki H, Hoshikawa H, et al. (1997) An endothelial receptor for oxidized low-density lipoprotein. Nature 386: 73-77. [Crossref]

19. Han KH, Tangirala RK, Green SR, Quehenberger O (1998) Chemokine receptor CCR2 expression and monocyte chemoattractant protein-1-mediated chemotaxis in human monocytes. A regulatory role for plasma LDL. Arterioscler Thromb Vasc Biol 18: 19831991. [Crossref]

20. Nagy L, Tontonoz P, Alvarez JG, Chen H, Evans RM (1998) Oxidized LDL regulates macrophage gene expression through ligand activation of PPARgamma. Cell 93: 229240. [Crossref]

21. Chawla A, Barak Y, Nagy L, Liao D, Tontonoz P, et al. (2001) PPAR-gamma dependent and independent effects on macrophage-gene expression in lipid metabolism and inflammation. Nat Med 7: 48-52. [Crossref]

22. Chawla A, Boisvert WA, Lee CH, Laffitte BA, Barak Y, et al. (2001) A PPAR gamma-LXR-ABCA1 pathway in macrophages is involved in cholesterol efflux and atherogenesis. Mol Cell 7: 161-171. [Crossref]

23. Ishii T, Itoh K, Ruiz E, Leake DS, Unoki H, et al. (2004) Role of Nrf2 in the regulation of $\mathrm{CD} 36$ and stress protein expression in murine macrophages: activation by oxidatively modified LDL and 4-hydroxynonenal. Circ Res 94: 609-616.

24. Evans RM, Barish GD, Wang YX (2004) PPARs and the complex journey to obesity Nat Med 10: 355-361. [Crossref]

25. Willson TM, Cobb JE, Cowan DJ, Wiethe RW, Correa ID, et al. (1996) The structureactivity relationship between peroxisome proliferator-activated receptor gamma agonism and the antihyperglycemic activity of thiazolidinediones. J Med Chem 39: 665-668. [Crossref]

26. Li AC, Brown KK, Silvestre MJ, Willson TM, Palinski W, et al.(2000) Peroxisome proliferator activated receptor gamma ligands inhibit development of atherosclerosis in
LDL receptor-deficient mice. J Clin Invest 106: 523-531. [Crossref]

27. Han KH, Chang MK, Boullier A, Green SR, Li A, et al.(2000) Oxidized LDL reduce monocyte CCR2 expression through pathways involving peroxisome proliferatoractivated receptor gamma. J Clin Invest 106: 793-802. [Crossref]

28. Takeda K, Akira S (2005) Toll-like receptors in innate immunity. Int Immunol 17: 1-14 [Crossref]

29. Xu XH, Shah PK, Faure E, Equils O, Thomas L, et al. (2001) Toll-like receptor-4 is expressed by macrophages in murine and human lipid-rich atherosclerotic plaques and upregulated by oxidized LDL. Circulation 104: 3103-3108. [Crossref]

30. Mütze S, Rudnik-Schöneborn S, Zerres K, Rath W (2008) Genes and the preeclampsia syndrome. J Perinat Med 36: 38-58. [Crossref]

31. Moslemizade N, Rafiei A, Yazdani F, Hosseini-khah Z, Yusefnezhad K (2011) The effect of magnesium sulfate on bleeding time and nitric oxide production in preeclamsia. $\mathrm{Pak}$ J Biol Sci 14: 106-111. [Crossref]

32. Irminger-Finger I, Jastrow N, Irion O (2008) Preeclampsia: a danger growing in disguise. Int J Biochem Cell Biol 40: 1979-1983. [Crossref]

33. Nilsson PM, Lurbe E, Laurent S (2008) The early life origins of vascular ageing and cardiovascular risk: the EVA syndrome. J Hypertens 26: 1049-1057. [Crossref]

34. De Kat AC, Broekmans FJ, Laven JS, Van Der Schouw Y.T (2015) Anti-Müllerian Hormone as a marker of ovarian reserve in relation to cardio-metabolic health: A narrative review. Maturitas 80: 251-257. [Crossref]

35. Barden AE, Beilin LJ, Ritchie J, Walters BN, Michael C (1999) Does a predisposition to the metabolic syndrome sensitize women to develop pre-eclampsia? J Hypertens 17 1307-1315. [Crossref]

36. Magnussen EB, Vatten LJ, Lund-Nilsen TI, Salvesen KA, Davey Smith G, et al. (2007) Prepregnancy cardiovascular risk factors as predictors of pre-eclampsia: population based cohort study. BMJ 335: 978. [Crossref]

37. Huxley RR, Shiell AW, Law CM (2000) The role of size at birth and postnatal catch-up growth in determining systolic blood pressure: a systematic review of the literature. $J$ Hypertens 18: 815-831. [Crossref]

38. Huxley R, Neil A, Collins R (2002) Unravelling the fetal origins hypothesis: is there really an inverse association between birthweight and subsequent blood pressure? Lancet 360: 659-665. [Crossref]

39. Bryson CL, Ioannou GN, Rulyak SJ, Critchlow C (2003) Association between gestational diabetes and pregnancy-induced hypertension. Am J Epidemiol 158: 11481153. [Crossref]

40. Margolis KL, Manson JE, Greenland P, Rodabough RJ, Bray PF, et al. (2005) Leukocyte count as a predictor of cardiovascular events and mortality in postmenopausal women: the Women's Health Initiative Observational Study. Arch Intern Med 165: 500-508. [Crossref]

41. Kuhnert M, Schmidt S (2000) Changes in lymphocyte subsets during pregnancy and post-partum in cases of beginning eclampsia. J Perinat Med 28: 389-398. [Crossref]

42. Friedman GD, Klatsky AL, Siegelaub AB (1974) The leukocyte count as a predictor of myocardial infarction. N Engl J Med 290: 1275-1278. [Crossref]

43. Friedman GD, Selby JV, Quesenberry CP Jr (1990) The leukocyte count: a predictor of hypertension. J Clin Epidemiol 43: 907-911. [Crossref]

Copyright: $\odot 2016$ Ababio GK. This is an open-access article distributed under the terms of the Creative Commons Attribution License, which permits unrestricted use, distribution, and reproduction in any medium, provided the original author and source are credited. 